\title{
Adherencia al tratamiento en personas con alteraciones cardiovasculares: Enfoques teóricos de enfermería
}

\section{Adherence to treatment in people with cardiovascular disorders: Nursing theoretical approaches}

\section{Adesão ao tratamento em pessoas com doenças cardiovasculares: abordagens teóricas da enfermagem}

\section{Jennifer Rojas Reyes ${ }^{1}$, Luisa Fernanda Moscoso Loaiza²}

${ }^{1}$ Candidata a PhD en Enfermería, Magíster en Enfermería, Enfermera. Universidad Nacional de Colombia, Facultad de Enfermería. Bogotá, Colombia. Correo electrónico:

\section{jrojasre@unal.edu.co}

${ }^{2}$ Doctora en Enfermería, Magíster en Enfermería, Enfermera. Docente Programa de Enfermería Fundación Universitaria Sanitas. Bogotá, Colombia. Correo electrónico:

\section{lfmoscosolo@unisanitas.edu.co}

Cómo citar este artículo en edición digital: Rojas Reyes, J. y Moscoso Loaiza, L.F. (2020). Adherencia al tratamiento en personas con alteraciones cardiovasculares: Enfoques teóricos de enfermería. Cultura de los Cuidados (Edición digital), 24 (56) Recuperado de http://dx.doi.org/10.14198/cuid.2020.56.18

Correspondencia: Avenida Carrera 30 No.45-03 Ciudad Universitaria Edificio 228, Piso 3 Of. 306. Bogotá, Colombia.

Correo electrónico de contacto: jrojasre@unal.edu.co

Recibido:06/11/2019

Aceptado:30/01/2020

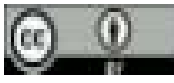

\section{ABSTRACT}

The aim of this article is to describe and analyze the theoretical approaches to nursing that have been used for research on adherence to treatment in people with cardiovascular diseases. For this, a descriptive integrative review is carried out between the years 2005 - 2018 in which 27 original articles are included, review, master's thesis and doctorate in nursing taking into account the descriptors adherence to treatment, nursing theories and cardiovascular health. As a result, 4 nursing theories that have been worked on in relation to adherence to treatment were identified: Deliberative Nursing Process Theory, Theory of Self-efficacy, Theory of Self-Care and Theory of Self-Care in Cardiac Failure; evidencing in literature the predominance of descriptive, methodological and intervention studies using these theoretical approaches. It can be concluded that the theoretical frameworks of nursing have allowed the investigative advance in the adherence of this population, however, with the evolution of knowledge it 
is required to explore other situational theoretical approaches or derived from the practice.

Keywords: Adherence to treatment, nursing theory, cardiovascular health, cardiovascular diseases, nursing research.

\section{RESUMEN}

El objetivo de este artículo es describir y analizar los enfoques teóricos de enfermería que han sido utilizados para la investigación sobre adherencia al tratamiento en personas con enfermedades cardiovasculares. Para ello, se realiza una revisión integrativa descriptiva entre los años 2005 - 2018 en el que se incluyen 27 artículos originales, de revisión, tesis de maestría y doctorado en enfermería teniendo en cuenta los descriptores adherencia al tratamiento, teorías de enfermería y salud cardiovascular. Como resultado, se identificaron 4 teorías de enfermería que más se han trabajado con relación a la adherencia al tratamiento: Teoría del Proceso de Enfermería Deliberativa, Teoría de la Autoeficacia, Teoría de Autocuidado y Teoría del autocuidado en Falla Cardíaca; evidenciando en la literatura predominio de estudios descriptivos, metodológicos y de intervención utilizando estos enfoques teóricos. Se puede concluir que los marcos teóricos de enfermería han permitido el avance investigativo en la adherencia de esta población, sin embargo, con la evolución del conocimiento se requiere explorar otros enfoques teóricos situacionales o derivados de la práctica.

Palabras clave: Adherencia al tratamiento, teoría de enfermería, salud cardiovascular, enfermedades cardiovasculares, investigación en enfermería.

\section{RESUMO}

O objetivo deste artigo é descrever e analisar as abordagens teóricas em enfermagem que vêm sendo utilizadas para pesquisas sobre adesão ao tratamento em pessoas com doenças cardiovasculares. 2018, que inclui 27 artigos originais, artigos de revisão, teses de mestrado e doutorado em enfermagem, considerando descritores de adesão ao tratamento, teorias de enfermagem e saúde cardiovascular - para isso, uma revisão integrativa descritiva a partir de 2005 é realizada. Como resultado, foram identificados 4 teorias de enfermagem que têm trabalhado mais sobre adesão ao tratamento: Teoria Deliberativo do Processo de Enfermagem, a teoria da auto-eficácia, teoria da auto-cuidado e auto-cuidado Teoria na Insuficiência Cardíaca; evidenciando na literatura a predominância de estudos descritivos, metodológicos e de intervenção utilizando essas abordagens teóricas. Podese concluir que os referenciais teóricos da enfermagem permitiram o avanço investigativo na adesão dessa população, porém, com a evolução do conhecimento, é necessário explorar outras abordagens teóricas situacionais ou derivadas da prática. Palavras-chave: Adesão ao tratamento, teoria de enfermagem, saúde cardiovascular, doenças cardiovasculares, pesquisa em enfermagem.

\section{INTRODUCCIÓN}

Las enfermedades cardiovasculares son la principal causa de mortalidad en todo el mundo, y de acuerdo con la Organización Mundial para la Salud (OMS), para el 2030, cerca de 23.3 millones de personas morirán por causa de cardiopatías y accidentes cerebro-vasculares, presentándose inclusive en enfermedades con factores de riesgo modificables, en el que su control puede disminuir esta cifra. De aquí, que la adherencia al tratamiento se haya convertido en un tema tan relevante para lograr el control de estas cifras, y la modificación de conductas nocivas para la salud cardiovascular (Organización Mundial de la Salud, 2016).

Para diversos autores (Bonilla, 2007; Munro, 2009; Salcedo y Gómez, 2014), la 
adherencia se define como la persistencia de la persona en practicar y mantener las conductas de salud deseables y es el resultado de la participación activa y el acuerdo entre el paciente y el profesional de la salud, ésta es influenciada por el significado de salud - enfermedad y el sentido de riesgo personal, así como el nivel socioeconómico, el apoyo a la toma de decisiones, la autoeficacia y las fuentes de información de la persona. Durante este proceso, el papel del profesional de enfermería es ayudarlo a mantener un comportamiento adherente dentro de las limitaciones y las circunstancias que le influyen (Avillarraga, 2010; Bowry et al., 2011).

Desde Enfermería, se han realizado diversos avances en la adherencia al tratamiento en población con enfermedad cardiovascular, es así que, el papel del enfermero, tiene que ver con ayudar a los individuos a que entiendan, adopten $\mathrm{y}$ mantengan prácticas que propendan por la disminución de riesgos; esto se evidencia en estudios como los de Conthe y Márquez (2010) y la Fundación Jordi Gol i Gurina (2014), quienes demostraron que la atención de los pacientes por profesionales de enfermería especialmente entrenadas da lugar a mayor adherencia. Es así que, por el importante rol que enfermería desempeña en la adherencia al tratamiento en personas con enfermedades cardiovasculares se hace relevante describir y analizar los enfoques teóricos propios de la disciplina desde los que se investiga e interviene en este fenómeno.

\section{MÉTODOS}

Se realizó una revisión integrativa descriptiva (Whittemore y Knafl, 2005) para dar respuesta a la pregunta: ¿Qué enfoques teóricos en enfermería han sido utilizados como base para la investigación de la adherencia al tratamiento en personas con enfermedades cardiovasculares? La búsqueda se realizó en las bases de datos de Embase, Science Direct, Pubmed, Medline, Academic Search Complete, PsycARTICLES, BVS, LILACS, Scielo, Scopus y en el Repositorio institucional de la Universidad Nacional de Colombia limitado entre los años 2004 y 2018 y en idiomas inglés, español y portugués. Se utilizaron los términos DeCS/MeSH de Treatment Adherence and Compliance, Medication Adherence, Cardiovascular Diseases y Nursing Theory, utilizando en las ecuaciones los operadores boleanos AND y OR.

En la búsqueda total se obtuvieron 5180 publicaciones entre artículos y tesis de maestría y/o doctorado en enfermería, de estas 2800 fueron eliminadas por ser duplicados o tener un abordaje cualitativo, ya que al utilizar enfoques teóricos disciplinares aplicados al fenómeno de la adherencia no competen o aplican estas metodologías investigativas. Se seleccionaron luego 27 documentos por utilizar explícitamente un enfoque teórico de enfermería y relacionarlo con el fenómeno 
Revista científica de la Asociación de Historia y Antropología de los Cuidados (Universidad de Alicante)

de la adherencia al tratamiento en salud cardiovascular. Posteriormente se realizó crítica utilizando los criterios de rigor en la investigación, se emplearon los criterios de validez interna, externa, de constructo y estadística (Hernández, Fernández y Baptista, 2014). La Figura 1 describe el proceso de selección de los documentos. Adicional se realiza un análisis de las teorías de acuerdo con Fawcett y Desanto (2013) para exponer los componentes de cada uno de los enfoques.

\section{RESULTADOS Y DISCUSIÓN}

Se determinaron como prevalentes 4 enfoques teóricos de enfermería bajo una misma visión del mundo las cuales se presentan en la Tabla 1.

\section{Teoría del proceso de enfermería deliberativa de Jean Orlando}

Este tipo de teoría, basada en la comunicación interpersonal, logra intervenir en el factor de adherencia al tratamiento correspondiente a los relacionados con el equipo o el sistema de asistencia en salud, ya que una buena relación con el paciente permite llegar a acuerdos y generar la confianza necesaria para cumplir la terapéutica asignada.

En el estudio de Sheldon y Ellington (2008), demostraron que la comunicación de enfermería afecta los resultados de salud del paciente, como la ansiedad, la adherencia a los tratamientos y la satisfacción con la atención. La teoría de Orlando describe las reacciones de las enfermeras ante el comportamiento de los pacientes como generadores de percepción, pensamiento $\mathrm{y}$ sentimiento para luego actuar. Un modelo de procesamiento de la información social describe los pasos en los procesos cognitivos utilizados para responder, sin olvidar que la relación con el paciente y su familia debe estar basada en la confianza, la continuidad, la accesibilidad, la flexibilidad y la confidencialidad, como aspectos que pueden potenciar la adherencia (Sotoca y Codina, 2006).

Para Herrero y Benavente (2016), la comunicación como la plantea Orlando es la vía básica para lograr cambiar el comportamiento no saludable del paciente, proporcionar información adaptada a las necesidades específicas y aumentar su participación en la toma de decisiones respecto a su salud, potencia la implicación del paciente en su propio tratamiento. En contraposición, el estudio de Rodríguez y Gómez (2010) evidenció que frente a tratamientos complejos en ausencia de guías o soporte social con el profesional de enfermería, llevan al abandono del tratamiento por no entender cómo proceder. Es aquí donde una comunicación efectiva puede rescatar los comportamientos adherentes farmacológicos y no farmacológicos del paciente con enfermedad cardiovascular y su familia. 


\section{Teoría de la Autoeficacia de Bárbara} Resnik

Los supuestos teóricos que hacen parte de este enfoque permiten manejar los factores personales de la adherencia para superar las dificultades que se encuentran en el entorno y el mismo sistema de salud (Resnick, 2003). Uno de estos aspectos personales es la motivación para adherirse al tratamiento, la cual depende del valor que el paciente le asigna a este comportamiento, por lo que acrecentar la motivación intrínseca de un paciente mediante el aumento de la importancia percibida de la adherencia y fortalecer la confianza, son metas del tratamiento comportamental que deben abordarse si se busca mejorar la adherencia (Aggarwal y Mosca, 2010).

Lo anterior es respaldado por varios estudios (Álvarez y Barra, 2010; Gutiérrez y Gómez, 2018; Ortiz y Ortega, 2011), que lograron demostrar que la autoeficacia percibida tiene una mayor capacidad predictiva de la adherencia al tratamiento (89\%), que las variables integradas en el modelo de creencias en salud (71\%), de manera que esta teoría ha sido utilizada en el diseño de intervenciones para lograr una óptima adherencia al manejar la motivación como estrategia para modificar la conducta (Achury, 2017; Andrade y Céspedes, 2017; Holguín et al., 2006).

Achury y Durán de Villalobos (2016) desarrollaron una intervención de enfermería basada en la entrevista motivacional para mejorar la adherencia al tratamiento en los pacientes con isquemia cardíaca. Este fue un estudio cuasiexperimental en el que se logró en el grupo experimental cambios estadísticamente significativos $(p<0.013)$ en lo que se refiere al manejo de las situaciones tensionantes, atribuyendo este resultado a la relación que existe entre el manejo de estas situaciones y el incremento de la autoeficacia derivados de la entrevista motivacional.

Para la medición de este fenómeno, Achury (2017) creó y validó el instrumento denominado «Cuestionario para medir la adherencia al tratamiento de pacientes con enfermedad cardiovascular», el cual esta basado en la autoeficacia y consta de 29 ítems con una comprensibilidad superior al 95\% y una validez de constructo realizada con análisis factorial del $57.36 \%$ de la varianza explicada y un alfa de Cronbach de 0.837. Por su parte, Andrade y Céspedes (2017) realizan un ajuste y validación al mismo, resultando en la reformulación de 38 ítems, con un análisis factorial exploratorio que reportó seis factores con una varianza explicada de 61.83\%, un alfa de Cronbach de 0.92 para la escala total y dimensional por encima de 0.80 .

Por otra parte, se pueden encontrar instrumentos derivados de la teoría, como la Self efficacy for Appropriate Medication Adherence Scale - Seams que permite evaluar la autoeficacia en cuanto al comportamiento de adhesión medicamentosa en individuos con bajo nivel 
Revista científica de la Asociación de Historia y Antropología de los Cuidados (Universidad de Alicante)

de escolaridad. Su aplicación ayuda a condicionantes como determinantes de este identificar situaciones específicas, relacionadas a las creencias del paciente sobre la percepción de su capacidad para comportamiento (Achury, 2007; Bonilla, adherirse, de esa forma, dirigir las acciones del profesional de la salud con el objetivo de fortalecer la autoeficacia para adhesión medicamentosa (Pedrosa y Rodrigues, 2016).

La teoría de la autoeficacia deja ver cómo las expectativas de autoeficacia, de resultado y la percepción del riesgo juegan un papel relevante en los comportamientos que adoptan las personas en relación con la salud; favoreciendo la intervención en quienes presentan conductas relacionadas con una baja adherencia y manteniendo intervenciones de soporte en los pacientes adherentes (Andrade y Céspedes, 2017; Pedrosa y Rodrigues, 2016).

\section{Teoría General del Autocuidado de Dorothea Orem}

Varios estudios han confirmado una fuerte correlación entre la capacidad de agencia de autocuidado y la adherencia al tratamiento, revelando que cuando la persona más se cuida a sí misma, tiene una mejor adherencia (Bastidas, 2007; Olivella, Bonilla y Bastidas, 2012; Orem, 1995; Wkinson y Whitehead, 2009), siendo así que la adherencia al tratamiento se convierte en un resultado deseado de autocuidado en el que convergen los factores básicos 2009).

Velandia y Rivera (2009) sustentan que la falta de adherencia se da por una interrelación de factores entre los cuales se encuentran algunos derivados de la persona y su entorno económico, cultural y social; otros derivados de la enfermedad en sí, del tratamiento farmacológico y sus características, así como los derivados de los servicios de salud y del personal que atiende al paciente. La adherencia comprende acciones de autocuidado complejas y su éxito requiere de procesos cognitivos de razonamiento y toma de decisiones reflexionadas.

Ofman, Pereyra y Stefani (2013), realizaron un estudio sobre los comportamientos de autocuidado y adherencia terapéutica por género en pacientes hipertensos, en el que lograron describir que en dimensiones como "Responsabilidad con la salud" y "Nutrición” las mujeres puntuaron más alto que los hombres, es decir, ellas consultaron a los profesionales de la salud con mayor frecuencia y tuvieron una alimentación más sana. En las dimensiones "Actividad física” y “Autorrealización” los hombres puntuaron más alto que las mujeres, lo que indica que realizaron más ejercicios físicos y fueron más decididos al concretar metas personales. 
Por su parte Hernández y Flórez (2015), basaron su trabajo en el sistema de apoyo educativo para diseñar una intervención de enfermería con Tecnologías de la información y la comunicación TICS para mejorar la adherencia al tratamiento en pacientes con insuficiencia cardíaca - IC -. Este fue un ensayo clínico controlado que tuvo dos grupos experimentales con seguimiento telefónico, mensajes de texto y correos electrónicos y un grupo control. Determinó que los grupos experimentales alcanzaron el rango de totalmente adheridos, mientras que el grupo control se mantuvo en frecuentemente adherido, demostrando diferencias estadísticamente significativas. La preferencia de los pacientes estuvo en el Seguimiento telefónico + mensajes de texto.

\section{Teoría del autocuidado en falla cardíaca}

Basados en la literatura y la práctica, Lee, Tkacs y Riegel (2009), determinaron que el mantenimiento efectivo de autocuidado (adherencia) y las prácticas de gestión de autocuidado (evaluación y gestión de síntomas) son complementarias a los tratamientos farmacológicos para retrasar la progresión de la IC y mejorar los resultados de salud (Conceição et al., 2015). Con el desarrollo de esta teoría situacional, se creó un instrumento denominado "Self-Care of Heart Failure Index" para evaluar los comportamientos de autocuidado de las personas con IC, con el fin de reconocer el mantenimiento, la gestión y la confianza del autocuidado, obteniendo una escala validada de 22 ítems (Riegel, Carlson y Moser, 2004; Riegel et al., 2009; Riegel et al., 2011).

Así, en México y Portugal realizaron una investigación psicométrica contextualizada con el fin de adaptar esta escala a su cultura. En México, Salcedo, Nava y Vega (2018), obtuvieron una escala de 18 ítems con un análisis factorial exploratorio KMO de 0,61 para 2 factores y un alfa de Cronbach de 0,73, evidenciando pruebas de validez y confiabilidad aceptables. En Portugal, Pinto Marques et al. (2016) determinaron la confiabilidad por alfa de Cronbach en cada subescala, dando resultados similares a la escala original (Mantenimiento de autocuidado: 0,64 vs 0,55- Gestión del autocuidado: 0,57 vs 0,59 - Confianza en el autocuidado: 0,84 vs $0,82)$.

Liou et al. (2015) estudiaron el efecto de un programa de autocuidado en pacientes con IC, por lo que realizaron un diseño cuasi-experimental en el que se midió el grado de conocimiento del paciente sobre la IC congestiva y el índice de autocuidado en falla cardíaca. La intervención basada en esta teoría, brindó una sesión de entrenamiento individual de autocuidado (reconocimiento de la IC, adherencia a medicamentos, dieta y evaluación de síntomas) y posteriormente tuvieron un seguimiento telefónico para monitorear el mantenimiento y gestión del autocuidado. El grupo experimental mostró una puntuación 
Revista científica de la Asociación de Historia y Antropología de los Cuidados (Universidad de Alicante)

media significativamente más alta en el conocimiento de IC que el grupo de control; el mantenimiento, la administración y la confianza en el autocuidado mejoraron significativamente después del seguimiento. Para Paradis et al. (2010), la teoría del autocuidado en falla cardíaca ayudó comprender los conceptos de autocuidado y elegir un modelo de intervención para evitar las complicaciones en estos pacientes, de manera que se realizó un estudio piloto para evaluar el efecto de una intervención con entrevista motivacional en las conductas de autocuidado de los pacientes con insuficiencia cardíaca, obteniendo resultados significativos con respecto a la confianza para la realización de conductas de autocuidado específicas para la IC ( $\mathrm{p}=$ $0,005)$ y frente al tamaño del efecto fue de 0,86 , es decir que la magnitud del efecto fue grande.

\section{CONCLUSIONES}

Los soportes teóricos identificados en esta revisión: Teoría del Proceso de Enfermería Deliberativa, Teoría de la Autoeficacia, Teoría del Autocuidado y Teoría del autocuidado en Falla Cardíaca, han guiado el cuidado y la investigación de enfermería en pacientes con enfermedades cardiovasculares, evidenciando que en esta población la adherencia al tratamiento depende de varios factores en los que se resalta, por un lado, la comunicación interpersonal entre la enfermera y el paciente, la cual permite llegar a acuerdos y generar la confianza necesaria para lograr identificar y cambiar el comportamiento no saludable del paciente, en donde, la motivación y el reconocimiento de la importancia de adherirse a un tratamiento, son fundamentales para generar el cambio. Este cambio genera en el paciente la puesta en marcha de acciones orientadas al mantenimiento de su salud, las cuales, al mantenerse en el tiempo, se potencian.

Los abordajes teóricos expuestos, se fundamentan principalmente en brindar herramientas cognitivas, que influyen en la toma de decisiones de manera reflexiva, de tal forma que se propicia el aumento, mejoría y mantenimiento de las conductas relacionadas con la adherencia a tratamientos farmacológicos y no farmacológicos, permitiendo el avance investigativo en la adherencia de esta población. Particularmente, los marcos teóricos de rango medio y situacionales han mostrado ser efectivos en la investigación y la práctica, su aplicación por medio de intervenciones específicas para promover comportamientos de adherencia logra modificar y mantener conductas saludables. Por lo que es necesario que estos principios sean considerados en el diseño y puesta en marcha de programas de promoción y prevención interdisciplinares, en los que el profesional de enfermería acompañe a las personas y sus familias en los procesos de transformación de estilos de vida. 
Las diferentes investigaciones incluidas en la revisión, muestran el avance en la inclusión de teorías para explicar y abordar fenómenos de la práctica de enfermería cardiovascular, en el marco de la adherencia, trascendiendo desde un abordaje descriptivo, hasta la creación de instrumentos que han sido validados, y la creación de algunas intervenciones, aspectos fundamentales para afianzar indicadores empíricos que expliquen y justifiquen los fenómenos de cuidado propuestos en cada una de las teorías.

Los marcos teóricos abordados han permitido el avance investigativo en la adherencia al tratamiento en personas con alteraciones cardiovasculares, sin embargo, la comprobación científica que se evidencia de su alcance práctico, es aún limitada. Por lo que se recomienda explorar otros enfoques teóricos, en donde tome más relevancia el contexto de la persona, se incluya la participación familiar, y a nivel investigativo se pueda realizar un seguimiento a largo plazo.

\section{BIBLIOGRAFÍA}

Achury-Beltrán, L.F. (2017). Validez y confiabilidad del cuestionario para medir la adherencia al tratamiento de pacientes con enfermedad cardiovascular. Aquichan, 17(4), 460-471. doi: DOI: 10.5294/aqui.2017.17.4.9

Achury-Beltrán, L.F., y Durán de Villalobos, M.M. (2016). La entrevista motivacional y la adherencia de los pacientes en pos infarto agudo de miocardio. (Tesis de Maestría). Bogotá: Facultad de Enfermería, Universidad
Nacional de Colombia. Recuperado de

http://bdigital.unal.edu.co/50920/1/53122219 .2015\%20\%281\%29.pdf

Achury-Saldana, D. (2007). Adherencia al tratamiento en paciente con falla cardíaca. Bogotá: Universidad Nacional de Colombia.

Aggarwal, B., y Mosca, L. (2010). Lifestyle and Psychosocial Risk Factors Predict Nonadherence to Medication. Ann. behav. med., 40, 228-233. DOI: 10.1007/s12160-0109212-6

Álvarez, E., y Barra, E. (2010). Autoeficacia, estrés percibido y adherencia terapéutica en pacientes hemodializados. Ciencia $y$ Enfermería, 26(3), 63-72. doi: 10.4067/S0717-95532010000300008

Andrade, A,. y Céspedes V. (2017). Adherencia al tratamiento en enfermedad cardiovascular: rediseño y validación de un instrumento. Enfermería Universitaria, 14(4), 266-276. doi: 10.1016/j.reu.2017.10.001.

Avillarraga, M. (2010). Determinación Social de la Adherencia Terapéutica: Alternativa conceptual y metodológica aplicada al caso de mujeres viviendo con VIH/SIDA. Bogotá: National Graphics Ltda.

Bastidas, C. (2007). Asociación entre la capacidad de agencia de autocuidado y la adherencia a tratamientos farmacológicos y no farmacológicos en personas con alguna condición de enfermedad coronaria. Av. Enferm., 25(2), 65-75. Recuperado de https://revistas.unal.edu.co/index.php/avenfer $\underline{\mathrm{m} / \text { article/view/12811/13630 }}$

Bonilla, C. (2007). Adherencia y factores que influyen en la adherencia al tratamiento farmacológico y no farmacológico en personas con riesgo cardiovascular. Revisión Literaria. Bogotá: Universidad Nacional de Colombia. 
Revista científica de la Asociación de Historia y Antropología de los Cuidados (Universidad de Alicante)

Bowry, A., Shrank, W.H., Lee, J.L., Stedman, M., \& Choudhry, N.K. (2011). A Systematic Review of Adherence to Cardiovascular Medications in Resource-Limited Settings. $J$ Gen Intern Med, 26(12), 1479-1491. DOI: 10.1007/s11606-011-1825-3.

Conceição, A.P., dos Santos, M.A., dos Santos, B. \& Diná Monteiro da Cruz, D.L. (2015). Autocuidado de los pacientes con insuficiencia cardíaca. Rev. Latino-Am. Enfermagem, 23(4), 578-86. doi: 10.1590/0104-1169.0288.2591

Conthe, P., y Márquez E. (2012). Una aproximación multidisciplinar al problema de la adherencia terapéutica en las enfermedades crónicas : estado de la situación y perspectivas de futuro. Madrid: Publicaciones SEMI. Recuperado de https://www.fesemi.org/publicaciones/semi/c onsensos/documento-de-consenso-unaaproximacion-multidisciplinar-al-problemade-la-adherencia

Fawcett, J. y Desanto, S. (2013). Contemporary nursing knowledge. $3^{\circ}$ ed. Philadelphia: F. A. Davis Company.

Fundación Jordi Gol i Gurina IDIAP. (2014). Efectividad de las estrategias para mejorar la adherencia terapéutica en pacientes crónicos: revisión meta-análisis. Madrid: IDIAP.

Guba, EG. (1981). Criteria for assessing the trustworthiness of naturalistic inquiries. ECTJ, 29, 75-91.

Gutiérrez, R., y Gómez A. (2018). Agencia de autocuidado y autoeficacia percibida en personas con cardiopatías isquémicas. Av.enferm., 36(2), 161 - 169. DOI: 10.15446/av.enferm.v36n2.65674

Hernández, C., y Flórez, M. (2015). Efectividad del uso de tecnologías de la información y la comunicación en la adherencia al tratamiento de los pacientes con insuficiencia cardiaca. (Tesis de Maestría). Bogotá: Facultad de Enfermería, Universidad Nacional de Colombia. Recuperado de http://bdigital.unal.edu.co/51099/1/52930556 .2015.pdf

Hernández, R., Fernández, C., y Baptista, M. (2014). Metodología de la investigación. $6^{\circ}$ edición. México: McGraw-Hill.

Herrero, L., y Benavente, M. (2016). La comunicación en la relación de ayuda. [Disertación]. Lima: Facultad Ciencias de la Salud, Universidad Pontificia de Salamanca: Perú.

Holguín, L., Correa, D., Arrivillaga, M., Cáceres, D,. y Varela, M. (2006). Adherencia al tratamiento de hipertensión arterial: Efectividad de un programa de intervención biopsicosocial. Univ. Psychol., 5(3), 535-547. Recuperado de https://revistas.javeriana.edu.co/index.php/re vPsycho/article/view/459

Lee, C.S., Tkacs, N.C. \& Riegel, B. (2009). The influence of heart failure self-care on health outcomes: Hypothetical cardioprotective mechanisms. J Cardiovasc Nurs., $\quad 24, \quad 179-187 . \quad$ doi: 10.1097/JCN.0b013e31819b5419.

Liou H.L., Chen H.I., Hsu S.C., Lee S.C., Chang C.J., \& Wu M.J. (2015). The effects of a self-care program on patients with heart failure. J Chin Med Assoc., 78(11), 648-56. doi: 10.1016/j.jcma.2015.06.004.

Marriner, A., \& Alligood, M. (2007). Modelos y teorías de enfermería. 6ta edición Madrid: Ed. Elsevier.

Méndez, R. (2009). Adherencia al tratamiento como comportamiento de Autocuidado en personas con factores de riesgo 
Cardiovasculares: una propuesta de cuidado. $A v$. enferm., 27(2), 25-32. Recuperado en https://revistas.unal.edu.co/index.php/avenfer m/article/view/12965/13999

Munro, S.H.. (2009). Concept Analysis of Adherence in the Context of Cardiovascular Risk Reduction. Nursing Forum, 44(1), 25-36. doi: 10.1111/j.1744-6198.2009.00124.x.

Ofman, S.D., Pereyra, C.I. \& Stefani, D. (2013). Autocuidado en pacientes hipertensos esenciales en tratamiento. Diferencias según género. Boletín de Psicología., (108), 91-106. Recuperado en https://www.uv.es/seoane/boletin/previos/N1 08-6.pdf

Olivella, M., Bonilla, C. y Bastidas, C. (2012). Fomento del autocuidado en la insuficiencia cardiaca. Enfermería Global, (25), 282 - 286. doi: 10.4321/S1695-61412012000100017

Orem, D. (1995). Nursing: Concepts of practice .5th ed. St. Louis: Mosby.

Organización Mundial de la Salud. (2016). Las 10 principales causas de defunción. Recuperado de http://www.who.int/es/newsroom/fact-sheets/detail/the-top-10-causes-ofdeath.

Ortiz, R., y Ortega, E. (2011). Capacidad predictiva de la adherencia al tratamiento en los modelos sociocognitivos de creencias en salud. Psicología y Salud, 21(1), 79-90. Recuperado https://www.uv.mx/psicysalud/psicysalud21-1/21-1/Godeleva-Rosa-Ortiz-Viveros.pdf

Paradis, V., Cossette, S., Frasure-Smith, N., Heppell, S., \& Guertin, M.C. (2010). The Efficacy of a Motivational Nursing Intervention Based on the Stages of Change on Self-care in Heart Failure Patients. $J$ Cardiovasc Nurs., 25(2), 130-141. doi: 10.1097/JCN.0b013e3181c52497.
Pedrosa, B.S,. y Rodrigues, C.M. (2016). Adaptation and evaluation of the measurement properties of the Brazilian version of the Self-efficacy for Appropriate Medication Adherence Scale. Rev. Latino-Am. Enfermagem. 24: e2692. DOI: 10.1590/15188345.0167.2692

Peterson, S. \& Bedrow, T. (2013). Middle range theories: application to nursing research. $3^{\circ}$ edition. Philadelphia, EEUU; Walters Kluwer.

Pinto, C., Lopes, M.J., Rebola, E. \& Pequito T. (2016). Self-care in Patients with Heart Failure. Revista Ibero-Americana de Saúde e Envelhecimento RIASE, 2(1), 409 - 420. doi: 10.24902/r.riase.2016.2(1).439

Resnick, B. (2003). The theory of self-efficacy. En: Smith M, Liehr P, ed. Middle range theory for nursing. New York: Springer publishing company.

Riegel, B. \& Vaughan, V. (2008). A SituationSpecific Theory of Heart Failure Self-care. $J$ Cardiovasc Nurs, 23(3), 190-196. doi: 10.1097/JCN.0000000000000244

Riegel, B., Carlson, B. \& Moser, D.K. (2004). Psychometric testing of the self-care of heart failure index. Journal of Cardiac Failure, 10(4), 350-360. doi: 10.1016/j.cardfail.2003.12.001

Riegel, B., Lee, C.S., Dickson, V.V., \& Carlson B. (2009). An update on the self-care of heart failure index. $J$ Cardiovasc Nurs., 24(6), 485-497. doi: 10.1097/JCN.0b013e3181b4baa0

Riegel, B., Lee, C.S., Albert, N., Lennie, T., Chung, M., \& Song, E.K. (2011). From novice to expert: confidence and activity status determine heart failure self-care performance. Nurs Res., 2011, 60(2), 132-8. 
Revista científica de la Asociación de Historia y Antropología de los Cuidados (Universidad de Alicante)

Rodríguez, A. y Gómez, A. (2010). Factores Wkinson, A., y Whitehead, L. (2009). influyentes en adherencia al tratamiento en pacientes con riesgo cardiovascular. $A v$. enferm., 28(1), 63-71. Recuperado de https://revistas.unal.edu.co/index.php/avenfer $\mathrm{m} /$ article/view/15656

Evolution of the concept of self-care and implications for nurses: a literature review. International Journal of Nursing Studies, 46(8), 1143-1147. doi: 10.1016/j.ijnurstu.2008.12.011.

Salcedo, A., y Gómez, A. (2014). Grados de riesgo para la adherencia terapéutica en personas con hipertensión arterial. Av. Enferm, 32(1), 33-43. doi: https://doi.org/10.15446/av.enferm.v32n1.46 $\underline{033}$

Salcedo, R.A., Nava, D.C., y Vega S. (2018). Construcción y validación de una escala de comportamientos de autocuidado para personas con insuficiencia cardiaca en población mexicana. Rev Enferm Inst Mex Seguro Soc., 26(2), 73-81. Recuperado de https://www.medigraphic.com/pdfs/enfermeri aimss/eim-2018/eim182c.pdf

Sheldon, L.K., \& Ellington, L. (2008). Application of a model of social information processing to nursing theory: how nurses respond to patients. J Adv Nurs, 64(4), 388-98. doi: 10.1111/j.1365-2648.2008.04795.x

Sotoca, J., y Codina, C. (2006). Cómo mejorar la adherencia al tratamiento Terapéutica. JANO, 28(1605), 39-40. Recuperado de www.doyma.es/jano.

Velandia, A., y Rivera L. (2009). Agencia de Autocuidado y Adherencia al Tratamiento en Personas con Factores de Riesgo Cardiovascular. Rev. salud pública, 11(4), 538-548. Recuperado de http://www.scielo.org.co/pdf/rsap/v11n4/v11 n4a05.pdf

Whittemore, R., \& Knafl, K. (2005). The integrative review: updated methodology. $J$ Adv Nurs, 52(5), 546-53. doi: 10.1111/j.13652648.2005.03621.x 
Figura 1. Diagrama de flujo de la startegia de búsqueda y selección de artículos

\section{Revisión de la literatura $\mathbf{n}=\mathbf{5 1 8 0}$}

Embase, Science direct, Pubmed, Medline, Academic search complete, PsycARTICLES, BVS, LILACS, Scielo, Scopus y Repositorio institucional

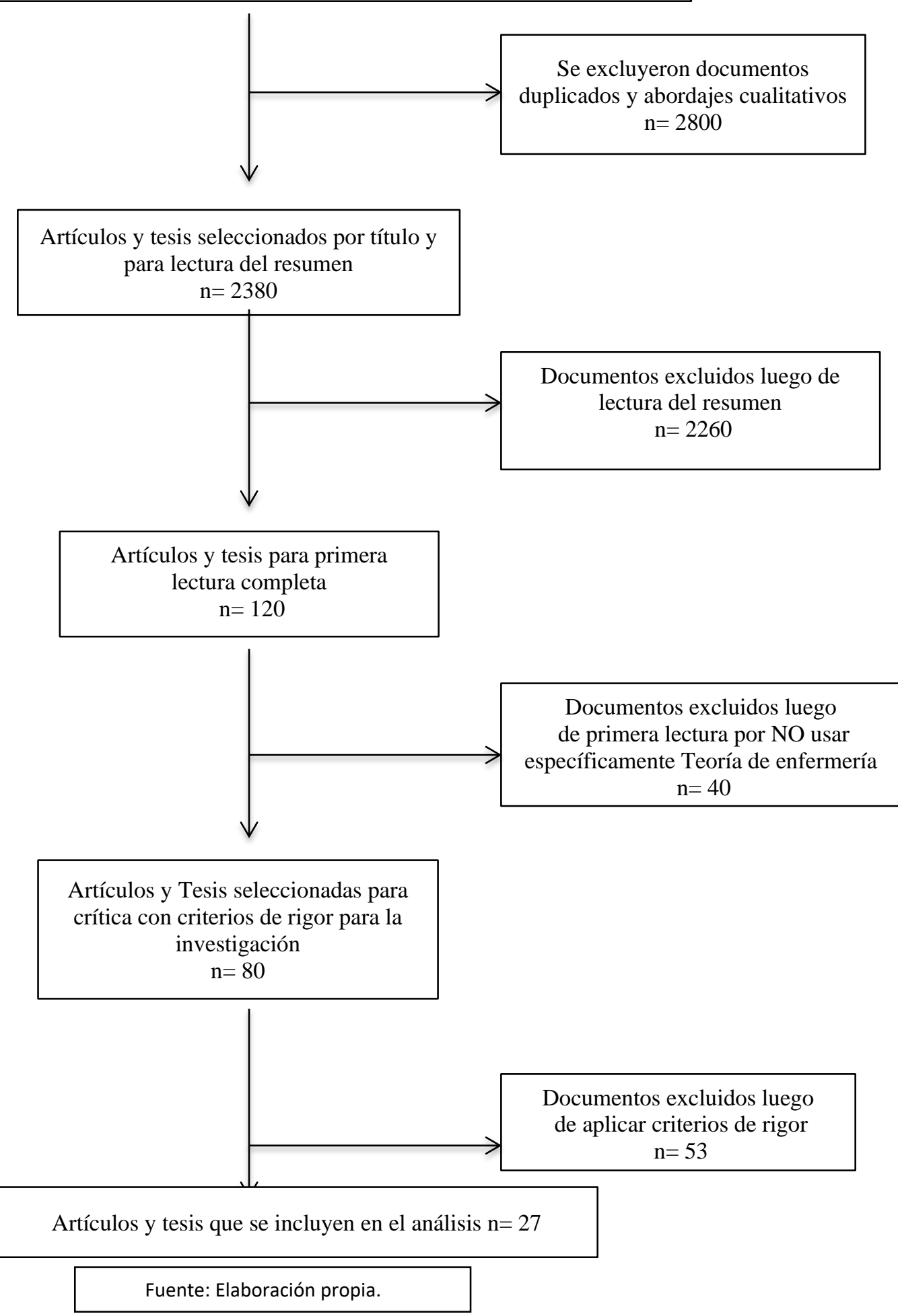




\section{Tabla 1. Descripción de cada marco teórico de enfermería según Fawcett y Desanto (2013)}

\begin{tabular}{|c|c|c|c|c|}
\hline $\begin{array}{c}\text { Componentes / } \\
\text { Teorías }\end{array}$ & $\begin{array}{l}\text { Teoría del proceso de } \\
\text { enfermería } \\
\text { deliberativa }\end{array}$ & $\begin{array}{ll}\text { Teoría de la } \\
\text { Autoeficacia }\end{array}$ & $\begin{array}{l}\text { Teoría } \\
\text { Autocuidado }\end{array}$ & $\begin{array}{l}\text { Teoría } \\
\text { autocuidado en falla } \\
\text { cardíaca }\end{array}$ \\
\hline $\begin{array}{c}\text { Artículos y/o } \\
\text { Tesis }\end{array}$ & $\begin{array}{l}\text { Diseños cuantitativos } \\
\text { descriptivos: } \\
\text { - Sotoca y Codina, } \\
2006 \\
\text { - Sheldon y Ellington, } \\
2008 \\
\text { - Herrero y Benavente, } \\
2016\end{array}$ & $\begin{array}{l}\text { Diseños psicométricos, } \\
\text { predictivos y } \\
\text { experimentales: } \\
\text { - Holguín et al., } 2006 \\
\text { - Aggarwal y Mosca, } \\
2010 \\
\text { - Álvarez y Barra, } 2010 \\
\text { - Ortiz y Ortega, 2011 } \\
\text { - Achury y Durán de } \\
\text { Villalobos, 2016 } \\
\text { - Pedrosa y Rodrigues, } \\
\text { 2016 } \\
\text { - Achury, 2017 } \\
\text { - Andrade y Céspedes, } \\
2017 \\
\text { - Gutiérrez y Gómez, } \\
2018\end{array}$ & $\begin{array}{l}\text { Diseños psicométricos, } \\
\text { predictivos y } \\
\text { experimentales: } \\
\text { - Achury, 2007 } \\
\text { - Bastidas, 2007 } \\
\text { - Bonilla, } 2007 \\
\text { - Méndez, 2009 } \\
\text { - Velandia y Rivera, } \\
\text { 2009 } \\
\text { - Wkinson } \\
\text { Whitehead, 2009 } \\
\text { - Olivella, Bonilla y } \\
\text { Bastidas, 2012 } \\
\text { - Ofman, Pereyra y } \\
\text { Stefani, 2013 } \\
\text { - Hernández y Flórez, } \\
\text { 2015 }\end{array}$ & $\begin{array}{l}\text { Diseños psicométricos } \\
\text { y experimentales: } \\
\text { - Lee, Tkacs y Riegel, } \\
\text { 2009 } \\
\text { - Conceição et al., } 2015 \\
\text { - Riegel, Carlson y } \\
\text { Moser, 2004 } \\
\text { - Riegel et al., } 2009 \\
\text { - Salcedo, Nava y Vega, } \\
\text { 2018 } \\
\text { - Liou et al., 2015 } \\
\text { - Paradis et al., 2010 } \\
\text { - Pinto Marques et al., } \\
\text { 2016 } \\
\text { - Riegel et al., 2011 }\end{array}$ \\
\hline $\begin{array}{l}\text { Orígenes y } \\
\text { Alcance } \\
\text { Visión } \\
\text { interactiva - } \\
\text { integrativa }\end{array}$ & $\begin{array}{l}\text { Teoría de rango medio } \\
\text { (TRM) descriptiva. } \\
\text { Tuvo su origen en la } \\
\text { observación de } \\
\text { interacciones enfermera } \\
\text { - paciente y su análisis } \\
\text { cualitativo. }\end{array}$ & $\begin{array}{l}\text { Teoría de rango medio } \\
\text { prescriptiva. Tuvo su } \\
\text { origen en la teoría social } \\
\text { cognitiva de Bandura. }\end{array}$ & $\begin{array}{l}\text { Teoría general. Tuvo su } \\
\text { origen en observaciones } \\
\text { del quehacer de las } \\
\text { enfermeras en el } \\
\text { cuidado de los pacientes } \\
\text { en todos los niveles de } \\
\text { atención. }\end{array}$ & $\begin{array}{l}\text { Teoría situacional. } \\
\text { Tuvo su origen en la } \\
\text { observación } \\
\text { personas } \\
\text { insuficiencia cardíaca y } \\
\text { el desarrollo de } \\
\text { instrumentos } \\
\text { específicos. }\end{array}$ \\
\hline
\end{tabular}




\begin{tabular}{|c|c|c|c|c|}
\hline $\begin{array}{l}\text { Definición } \\
\text { de } \\
\text { Enfermería }\end{array}$ & $\begin{array}{l}\text { Es una profesión que } \\
\text { actúa de forma } \\
\text { autónoma. } \\
\text { preocupa de una } \\
\text { necesidad de ayuda } \\
\text { real o potencial de un } \\
\text { individuo en una } \\
\text { circunstancia } \\
\text { inmediata y utiliza la } \\
\text { interacción } \\
\text { enfermera-paciente } \\
\text { para aliviar el } \\
\text { sufrimiento } \\
\text { paciente del } \\
\text { aumentar su angustia. }\end{array}$ & $\begin{array}{lr}\text { La autora de la teoría } \\
\text { no la } & \text { define } \\
\text { explícitamente. } & \text { Sin } \\
\text { embargo, } & \text { puede } \\
\text { inferirse. } & \\
\text { Enfermería debe } \\
\text { acompañar y motivar a } \\
\text { los individuos } \\
\text { partiendo de su propia } \\
\text { experiencia de salud } \\
\text { para modificar su } \\
\text { comportamiento a uno } \\
\text { más saludable. }\end{array}$ & $\begin{array}{l}\text { Es el servicio humano, que se } \\
\text { presta cuando la persona no puede } \\
\text { cuidarse por sí misma para } \\
\text { mantener la salud, la vida y el } \\
\text { bienestar. Es proporcionar a las } \\
\text { personas asistencia directa en su } \\
\text { autocuidado, según sus } \\
\text { requerimientos y de acuerdo a la } \\
\text { valoración. }\end{array}$ & $\begin{array}{lr}\text { La autora de la teoría } \\
\text { no la } & \text { define } \\
\text { explícitamente. Sin } \\
\text { embargo, } \\
\text { inferirse. } \\
\text { Enfermería } \\
\text { apoyar a la persona } \\
\text { para debe } \\
\text { conocimiento desarrollar } \\
\text { habilidad en el manejo } \\
\text { de los síntomas, } \\
\text { contribuyendo a la } \\
\text { adecuada toma de } \\
\text { decisiones. }\end{array}$ \\
\hline $\begin{array}{c}\text { Propósito o } \\
\text { Meta }\end{array}$ & $\begin{array}{l}\text { Mediante la relación } \\
\text { paciente - enfermera } \\
\text { se espera mejorar la } \\
\text { conducta del paciente } \\
\text { mediante el alivio de } \\
\text { su angustia, } \\
\text { produciendo un } \\
\text { cambio positivo. }\end{array}$ & \begin{tabular}{lr}
\multicolumn{3}{l}{ Potenciar la capacidad } \\
de las personas para \\
influir sobre su \\
comportamiento a \\
través del pensamiento \\
reflexivo y otras \\
herramientas r de \\
autoinfluencia.
\end{tabular} & $\begin{array}{l}\text { Establecer un modelo de suplencia } \\
\text { donde el rol de la enfermera } \\
\text { consiste en ayudar a realizar } \\
\text { acciones que la persona no puede } \\
\text { llevar a cabo por si solas. }\end{array}$ & $\begin{array}{llr}\text { Lograr que los } & \text { lon } \\
\text { pacientes como los } \\
\text { principales } & \text { actores en } \\
\text { decisiones de salud, } & \text { puedan tener las } \\
\text { herramientas para } \\
\text { manejar sus problemas } \\
\text { de con la falla cardíaca. }\end{array}$ \\
\hline $\begin{array}{l}\text { Conceptos y } \\
\text { supuestos } \\
\text { teóricos }\end{array}$ & 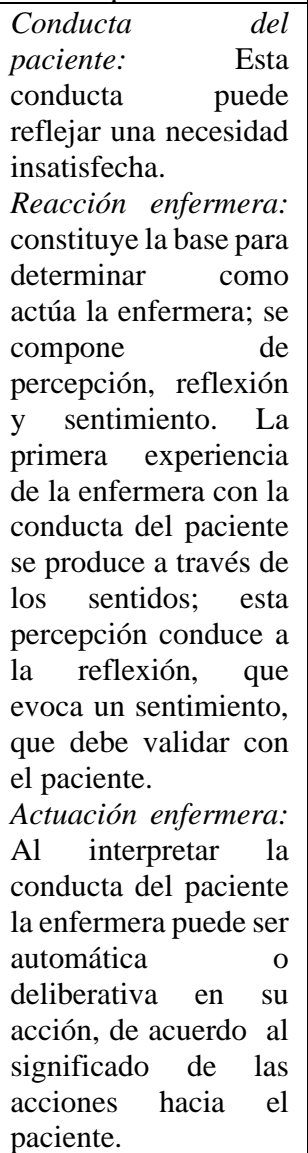 & $\begin{array}{l}\text { Expectativas de } \\
\text { autoeficacia: los } \\
\text { juicios acerca de la } \\
\text { capacidad personal } \\
\text { para realizar una tarea } \\
\text { determinada. } \\
\text { Expectativas de } \\
\text { resultados: los juicios } \\
\text { sobre lo que sucederá, } \\
\text { si se lleva a cabo con } \\
\text { éxito una tarea } \\
\text { determinada. } \\
\text { Autoeficacia } \\
\text { percibida: los juicios } \\
\text { de cada individuo sobre } \\
\text { sus capacidades. } \\
\text { Características: } \\
\text { - Modelado: El éxito } \\
\text { continuado refuerza la } \\
\text { persistencia. } \\
\text { - Experiencia vicaria: } \\
\text { Viendo a otras } \\
\text { personas tener éxito, el } \\
\text { sujeto puede pensar } \\
\text { que él también puede. } \\
\text { - Persuasión verbal: } \\
\text { No es un simple elogio. } \\
\text { - Retroalimentación: } \\
\text { informan sobre las } \\
\text { competencias. }\end{array}$ & 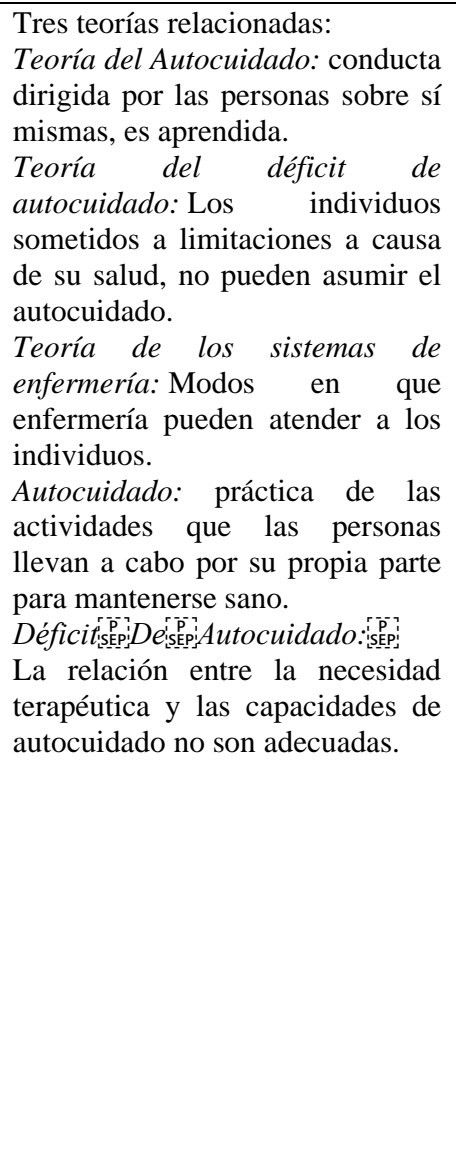 & 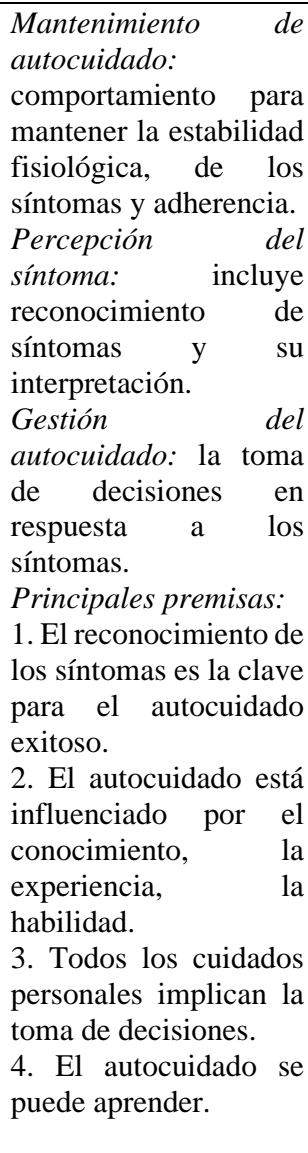 \\
\hline
\end{tabular}

